\title{
Microscale heterogeneity of soil bacterial communities under long-term fertilizations in fluvo-aquic soils
}

\author{
Haojie Feng ${ }^{\dagger}$, Hong Pan ${ }^{\dagger}$, Chengliang Li $i^{*}$, Yuping Zhuge* \\ National Engineering Laboratory for Efficient Utilization of Soil and Fertilizer Resources, College of Resources and Environment, \\ Shandong Agricultural University, Taian 271018, China
}

H I G H L I G H T S

- Both organic and inorganic fertilizations stimulate soil aggregation.

- Organic and inorganic fertilizers enhance organic carbon storage at aggregate scale.

- Aggregate-associated bacterial communities were more sensitive to organic fertilizers than to chemical ones.

- The complexity of bacterial network structures decreased with decreasing of aggregate size.

- The competitive interactions among bacterial communities were intensified with decreasing of aggregate size.

\section{ARTICLE INFO}

Article history:

Received July 4, 2021

Revised August 25, 2021

Accepted September 14, 2021

Keywords:

Bacterial community

Soil aggregate

Long-term fertilization

Soil organic carbon (SOC)
GRAPHICAL ABSTRACT

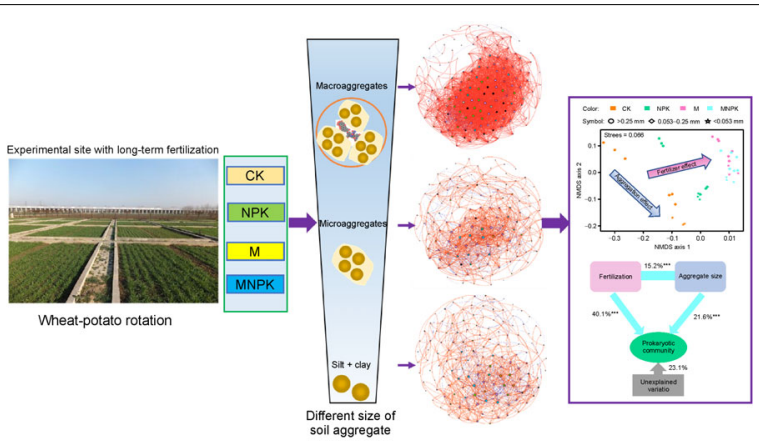

\section{A B S T R A C T}

Differently sized soil aggregates, with non-uniform distribution of space and nutrients, provide spatially heterogeneous microenvironments for microorganisms and are important for controlling microbial community ecology and biogeochemistry in soils. Here, we investigated the prokaryotic communities within different aggregate-size fractions: macroaggregate $(>0.25 \mathrm{~mm})$, microaggregate $(0.053-0.25 \mathrm{~mm})$ and silt + clay $(<0.053 \mathrm{~mm})$. These were isolated from fluvo-aquic soils under 39-year fertilization strategies: no fertilizer (CK), chemical fertilizer (NPK), manure fertilizer (M), and combination of manure and chemical fertilizers (MNPK). The results showed that the proportion of macroaggregate, soil aggregate-associated organic carbon (SOC) content and aggregate stability were all significantly increased by both manure and chemical fertilizations. Organic fertilizations (M and MNPK) more effectively boosted formation and stability of macroaggregates and enhanced SOC concentration than NPK. The distribution patterns of microorganisms in aggregates were primarily shaped by fertilization and aggregate size. They explained $76.9 \%$ of the variance in bacterial community compositions. Fertilizations, especially with organic fertilizers primarily transitioned bacterial communities from slow-growing oligotrophic groups (e.g., Chloroflexi) dominance to fast-growing copiotrophic groups (e.g., Proteobacteria and Bacteroidetes) dominance across all aggregate sizes. Macroaggregates possessed a more stable bacterial community and efficiency of resource transfer, while smaller aggregates increased antagonism and weakened mutualism among bacterial communities. Overall, combination of manure and chemical fertilizers was crucial for increasing SOC content and aggregation, leading to a clear shift in bacterial community structures at aggregate scale.

(c) Higher Education Press 2021

\section{Introduction}

* Corresponding authors

E-mail address: chengliang_li11@163.com (C. Li); zhugeyp@sdau.edu.cn (Y. Zhuge)

These authors contributed equally to this work.
Soil particles are classified as of sand, silt and clay, and these can bind into aggregates of different sizes and stability due to action of organic and inorganic binding agents (Briar et al., 
2011). Soil aggregates, with complex spatial and nutritional heterogeneity, are the principal soil structural units that are fundamental for soil quality and ecosystem functions. The distribution and stability of soil aggregates, and of the pores within and between them, affect many physical, chemical and biological processes in soils. Soil aggregates of various sizes and stability offer spatially heterogeneous microhabitats with organic matter stability, water potential, oxygen flux and predation pressure (Rillig et al., 2017). These variables affect the transportation of nutrient, transformation of element, diversity and activity of microbes (Six et al., 1999; Young et al., 2008; Muruganandam et al., 2010; Briar et al., 2011; Bailey et al., 2013; Fierer, 2017). Therefore, soil analysis at microscale levels can provide a fine and subtle picture of soil physicochemical and biological characteristics.

Previous studies reported that macroaggregates ( $>0.25 \mathrm{~mm}$ ) could foster a protective environment for soil organic matter (SOM), reduce SOM decomposition and favor SOM accumulation (Ashagrie et al., 2007; Lin et al., 2019). An increase in SOM in turn could enhance formation and stabilization of aggregates. The differences in physicochemical and structural characteristics in various aggregate sizes are likely to be the major factors shaping microbial community patterns (Liao et al., 2020). Meanwhile, soil microbes play critical roles in essential processes, such as cycling of nitrogen and phosphorus, decomposition and restoration of soil carbon, development and maintenance of soil aggregation (Jiang et al., 2017; Pan et al., 2020). Compared with smaller aggregates, macroaggregates were reported to serve as hotspots of microbial activity (Gupta and Germida, 2015) and show higher path tortuosity, effective porosity and easily accessible carbon, which favored $r$-selected bacterial species, like Proteobacteria and Actinobacteria (Liu et al., 2020). Microaggregates $(0.053-0.25 \mathrm{~mm})$ and silt + clay $(<0.053$ $\mathrm{mm}$ ) fractions, on the other hand, showed less complexity of pore network and higher recalcitrant carbon, which facilitated the growth of $k$-selected microbes, like Chloroflexi and Acidobacteria (Lin et al., 2019). Previous results revealed that methanotrophic related bacteria and Actinobacteria tended to inhabit larger aggregates, while some saprophytic and oligotrophic bacteria were abundant in smaller aggregates (Lin et al., 2019). Therefore, investigation of microbial compositions at microscale (such as in soil aggregates) is crucial for unravelling the bioavailability and cycling of biologically and biochemically mediated nutrients in soils.

Numerous studies documented that fertilization, an indispensable agricultural practice for enhancing soil nutrient and crop productivity, could decisively influence the spatial heterogeneity of aggregates (Han et al., 2020; Liu et al., 2020), which may affect the succession of soil microbial groups involved in given biogeochemical processes (Han et al., 2020). It is widely recognized that long-term manure and $\mathrm{NPK}+$ straw incorporation improved soil aggregation and structural stability and induced higher $\mathrm{C}$ content in macroaggregates (Wang and Yang, 2003; Zhou et al., 2013; Bottinelli et al., 2017; He et al., 2018a; Ghosh et al., 2019; Li et al., 2019), while some reports proposed that long-term manure amendments increased the macroaggregate dispersion and thus resulted in a decrease in aggregate stability (Whalen and Chang, 2002). Likewise, it has been reported that inorganic fertilizer alone could improve, deteriorate or exert no effect on soil structure (Stone et al., 1991; Biederbeck et al., 1996; Wang and Yang, 2003; Blanco-Canqui and Schlegel, 2013; Zhou et al., 2013; Jiang et al., 2017; Mustafa et al., 2020). Therefore, the influence of organic versus inorganic fertilization on soil aggregation needs further research.

The effects of fertilization strategies on soil microbial community patterns were greater in macroaggregates than those in smaller aggregates, as the microenvironment in macroaggregate were vulnerability (Tiemann et al., 2015; Trivedi et al., 2015; 2017). Meanwhile, macroaggregate usually showed a greater complexity of prokaryotic community network than microaggregate and silt + clay fractions, which suggested a higher community stability and efficiency of resource transfer (Neutel et al., 2007; Mougi and Kondoh, 2012; Morriën et al., 2017). Overall, aggregate size, rather than fertilization was found to have a stronger role to play in bacterial community structures (Liao et al., 2020). However, some studies found that fertilizer addition accompanied with a sharp increase of available nutrient could more easily change bacterial community than aggregate size (Jiang et al., 2013; Jiang et al., 2014; Han et al., 2018; Lin et al., 2019). A deep understanding of the fertilization effects on soil aggregation and associated prokaryotic communities is therefore indispensable for achieving better management of soil fertility.

Therefore, soils from a long-term field trial, initiated in 1980, with combined organic and inorganic fertilizations, were selected to identify the effects of long-term fertilizations on soil aggregate stability and microbiological properties at aggregate scale. We hypothesized the following: (i) both organic and inorganic fertilization could enhance aggregations and boost SOC stock; (ii) effects of fertilization on prokaryotic communities could be greater than those of aggregate size in agricultural soils under long-term fertilization; (iii) bacterial communities at aggregate scale were more sensitive to organic fertilizations than to inorganic ones. Understanding the allocation of prokaryotic communities within soil aggregate size fractions and their response to long-term fertilization and aggregate size change, induced by fertilization at the micron scale, would be helpful in revealing the underlying mechanism of how fertilization benefits soil quality, and would shed light on the development of effective fertilization regimes.

\section{Materials and methods}

\subsection{Study site and soil sampling}

The study site and field experiment have been described in detail previously by Pan et al. (2020). Briefly, the static 
fertilization experiment at the experimental farm of the Xuzhou Institute of Agricultural Sciences of the Xuhuai District, Jiangsu Province, China (117 ${ }^{\circ} 17^{\prime} \mathrm{E}$ and $34^{\circ} 16^{\prime} \mathrm{N}$ ) was initiated in 1980 . This field was subjected to a winter wheat-summer maize (Zea mays L.) rotation during 1980 2002, and a winter wheat (Triticum aestivum L.)-sweet potato rotation has been used since 2002 . The aboveground crop residues were baled and removed from the plots after harvest. The soil was classified as a fluvo-aquic soil with a sandy loam texture. The main characteristics of the initial soil were as follows: $10.8 \mathrm{~g} \mathrm{~kg}^{-1}$ soil organic matter (SOM), $0.66 \mathrm{~g} \mathrm{~kg}^{-1}$ total nitrogen (TN), $0.74 \mathrm{~g} \mathrm{~kg}^{-1}$ total phosphorus (TP), $12.0 \mathrm{mg} \mathrm{kg}^{-1}$ available phosphorus (AP), $63.0 \mathrm{mg} \mathrm{kg}^{-1}$ available potassium (AK) and a $\mathrm{pH}$ value of 8.01 .

Four treatments with three replicates were arranged in a randomized plot design including: non-fertilization control (CK); chemical NPK fertilizer (NPK); animal manure fertilizer (M); chemical NPK fertilizer combined with animal manure (MNPK). The annual application rate for the chemical fertilizer was $300 \mathrm{~kg} \mathrm{~N}$ ha ${ }^{-1}, 150 \mathrm{~kg} \mathrm{P}_{2} \mathrm{O}_{5} \mathrm{ha}^{-1}$, and 225 $\mathrm{kg} \mathrm{K}_{2} \mathrm{O} \mathrm{ha}{ }^{-1}$, in the form of urea $(46 \% \mathrm{~N})$, diammonium phosphate $\left(15 \% \mathrm{~N}, 42 \% \mathrm{P}_{2} \mathrm{O}_{5}\right)$ and potassium sulfate $(50 \%$ $\mathrm{K}_{2} \mathrm{O}$ ), respectively. Annual manure fertilizer was applied as horse compost during 1981-1984, and cattle compost since 1985.

Soil samples were collected in June 2019, when the winter wheat was harvested. Five intact soil samples were taken randomly in each plot at a depth of $0-20 \mathrm{~cm}$ and then pooled together to obtain a representative sample of about $2 \mathrm{~kg}$. The samples were immediately wrapped in ice packs and transported to the laboratory without any extrusion. The fresh samples were gently broken along the natural planes of weakness and were passed through an $8 \mathrm{~mm}$ sieve and then were split into two subsamples. One was stored at $4^{\circ} \mathrm{C}$ for determining soil physical-chemical properties, and one for aggregation fractionation (within one week). Variables that were identified for determination of soil properties, including $\mathrm{pH}, \mathrm{TN}, \mathrm{NH}_{4}{ }^{+}-\mathrm{N}, \mathrm{NO}_{3}{ }^{-}-\mathrm{N}$, Olsen $\mathrm{P}, \mathrm{AK}$ and SOM were detailed in Pan et al. (2020).

\subsection{Soil aggregate fractionation}

Water-stable aggregates were separated through a wetsieving method according to Elliott (1986) and comprised three size fractions: $>0.25 \mathrm{~mm}$ (macroaggregates), 0.25 $0.053 \mathrm{~mm}$ (microaggregates) and $<0.053 \mathrm{~mm}$ (silt + clay) (Lin et al., 2019; Han et al., 2021). The separated fractions were divided into two subsamples, one was freeze-dried and was stored at $-80^{\circ} \mathrm{C}$ for subsequent DNA extraction. The other subsample was used to determine soil aggregate organic carbon.

Soil water-stable aggregates (WSA) and mean weight diameter (MWD) were calculated as the indices of aggregate stability as described in Feng et al. (2019).

\subsection{DNA extraction and Illumina MiSeq sequencing}

A DNA sample from each soil aggregate fraction was extracted by FastDNA SPIN kit for soil (MP Biomedicals, LLC, Solon, OH, USA) according to the manufacturer's protocols. The DNA quantity and integrity were checked by a NanoDrop® ND-2000 UV-Vis Spectrophotometer (NanoDrop Technologies, Wilmington, DE, USA) and electrophoresis on a $0.7 \%$ agarose gel. The extracted DNA was stored at $-20^{\circ} \mathrm{C}$ until analysis.

The V4 hypervariable regions of the bacteria 16S rRNA gene were amplified with primers 515F (5'-GTGYCAGCMGCCGCGGTAA-3') and 806R (5'-GGACTACNVGGGTWTCTAAT-3') on a GeneAmp® 9700 (Applied Biosystems). The $\mathrm{PCR}$ reactions were conducted using the following program: $3 \mathrm{~min}$ of denaturation at $95^{\circ} \mathrm{C}, 27$ cycles of $30 \mathrm{~s}$ at $95^{\circ} \mathrm{C}, 30 \mathrm{~s}$ for annealing at $55^{\circ} \mathrm{C}$, and $45 \mathrm{~s}$ for elongation at $72^{\circ} \mathrm{C}$, and a final extension at $72^{\circ} \mathrm{C}$ for $10 \mathrm{~min}$. PCR reactions were performed in triplicate $20 \mu \mathrm{L}$ mixture containing $10 \mu \mathrm{L} 2 \times$ EasyTaq ${ }^{\circledR}$ PCR SuperMix (+ dye) (TransGen Biotech, Beijing, China), $1 \mathrm{mM}$ of each primer, $10 \mathrm{ng}$ of 10 -fold diluted DNA template, and 7.0-8.6 $\mu \mathrm{L}$ milli-Q water.

The resulting PCR products were checked by electrophoresis on a $2 \%$ agarose gel and further purified using the AxyPrep DNA Gel Extraction Kit (Axygen Biosciences, Union City, CA, USA) according to the manufacturer's instructions. Purified amplicons were pooled in equimolar and paired end sequenced $(2 \times 300)$ on an Illumina MiSeq platform (Illumina, San Diego, USA).

Raw fastq files were demultiplexed, quality-filtered by Trimmomatic and merged by FLASH with the following criteria: (i) The reads were truncated at any site receiving an average quality score $<20$ over a 50 bp sliding window. (ii) Primers were exactly matched allowing 2 nucleotide mismatching, and reads containing ambiguous bases were removed. (iii) Sequences whose overlap longer than $10 \mathrm{bp}$ were merged according to their overlap.

Operational taxonomic units (OTUs) were clustered with 97\% similarity cutoff using UPARSE (version $7.1 \mathrm{http}: / /$ drive 5 . com/uparse) and removed using UCHIME. The taxonomy of each 16S rRNA gene sequence was analyzed by RDP Classifier algorithm (http://rdp.cme.msu.edu/) against the Silva (SSU123) 16S rRNA database using confidence threshold of $70 \%$. Raw sequence data was deposited in the sequence read archive at the NCBI (https://www.ncbi.nlm. nih.gov/) under accession number PRJNA720043.

\subsection{Data and statistical analysis}

The means of soil properties, aggregate size, WSA, MWD among treatments, SOC among treatments and aggregate sizes were compared by One-way ANOVA with Duncan using SPSS software, version 20 (IBM Corp., Armonk, NY, USA). Non-metric multidimensional scaling (NMDS) analysis was performed based on Bray-Curtis distances. Permutational 
multivariate analysis of variance (PERMANOVA) was calculated to test and quantitatively determine the substantial effects of fertilization and soil aggregate size on the prokaryotic community structure using the adonis function. Both NMDS and PERMANOVA were analyzed in the vegan package of $R$ software, version 4.0.4. Circos graphs for the prokaryotic community patterns were developed using the online Circos software (http://www.circos.ca/). Linear discriminant analysis (LDA) effect size (LEfSe) was used to estimate which microbiome attributes (at all taxonomic levels) differed significantly by fertilizations at aggregate scales. Only taxa meeting a threshold for the LDA score for discriminate features of 3.5 were shown. Co-occurrence networks were constructed using the Spearman approach, estimating correlation values from OTU data. The OTUs with a summed relative abundance less than $0.1 \%$ were removed. The analysis was performed with statistically significant level $(P<0.05)$ and an absolute value over 0.75 using the "igraph" and "psych" packages in the R environment. The networks were visualized by the Fruchterman Reingold layout on the Gephi platform (0.9.2) (Bastian et al., 2009).

\section{Results}

\subsection{Aggregate size distribution and attributes}

The $<0.053 \mathrm{~mm}$ aggregates were dominant for all soils, accounting for $65.89 \%$ in MNPK-treated soils to $85.31 \%$ in soils without fertilization (Fig. 1A). Inorganic and organic fertilizers substantially impacted the distribution of the soil aggregate compared to the CK treatment. The proportions of macroaggregates and microaggregates were significantly increased by all fertilizer additions, especially by the application of manure in combination with chemical fertilizer (MNPK). In stark contrast, the proportion of $<0.053 \mathrm{~mm}$ silt + clay was decreased by all fertilizations, especially by MNPK treatment.

SOC exhibited clear differences $(P<0.01)$ among aggregate-size fractions and among fertilization regimes (Fig. 1B).
The content of SOC was increased with the increasing aggregate size under all fertilization practices. Fertilizations, especially organic fertilizer (M and MNPK) pronouncedly stimulated SOC content across different aggregate sizes. The SOC contents in the bulk soils ranged from $10.01 \mathrm{~g} \mathrm{~kg}^{-1}$ in CK soils to $16.11 \mathrm{~g} \mathrm{~kg}^{-1}$ in MNPK soils, and were notably increased by organic fertilizations ( $M$ and MNPK) and changed little by inorganic fertilizations (NPK).

The aggregate stability (WSA and MWD) was greater in fertilizer-treated soils than in CK soils (Fig. 1C). Both WSA and MWD were the highest in MNPK-treated soils, followed by Nand M-treated soils, which were significantly higher than $\mathrm{CK}$ soils.

\subsection{Soil bacterial compositions in soil aggregates}

Proteobacteria formed the most dominant phylum in all aggregates, followed by Acidobacteria, Planctomycetes, Bacteroidetes, Chloroflexi, Actinobacteria, Gemmatimonadetes, Verrucomicrobia, Rokubacteria, and Latescibacteria (Fig. 2). Together, these phyla accounted for $95.04 \%-97.23 \%$ in macroaggregates, $95.67 \%-97.26 \%$ in microaggregates, and $96.18 \%-97.08 \%$ in silt + clay fractions. The proportion of Proteobacteria was remarkably higher in macroaggregates than in smaller aggregates, and that of Chloroflexi was appreciably higher in macroaggregates and microaggregates than those in silt + clay. The relative abundance of Acidobacteria was meaningfully decreased by fertilizations in macroaggregate and microaggregate fractions compared with CK treatment. The proportions of Proteobacteria and Bacteroidetes were clearly increased, while those of Acidobacteria, Chloroflexi and Gemmatimonadetes were remarkably decreased by organic and inorganic fertilization treatments compared to CK treatment in all aggregates.

NMDS analysis revealed that the prokaryotic communities of fertilized soil aggregates were clearly distinguishable from the CK soil aggregates along the horizontal axis, while that of macroaggregates were obviously separated from microaggregates and silt + clay along the vertical axis (Fig. $3 \mathrm{~A})$. Of
A

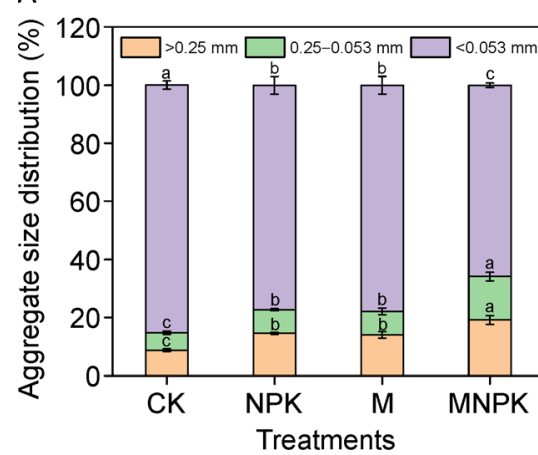

B

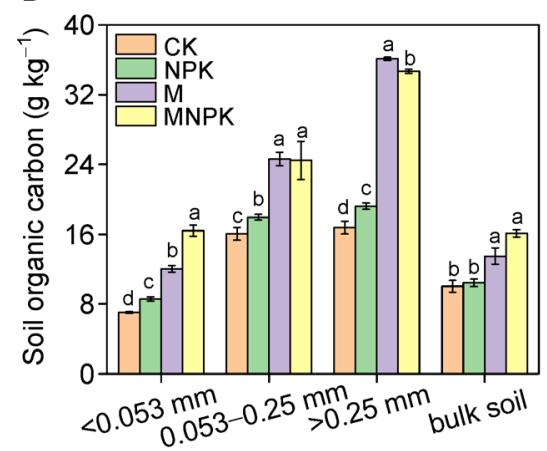

C

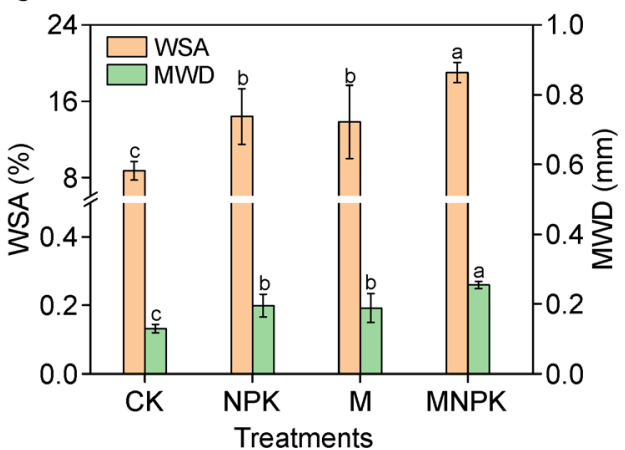

Fig. 1 Proportion of macroaggregates ( $>0.25 \mathrm{~mm})$, microaggregates $(0.053-0.25 \mathrm{~mm})$ and silt + clay sized fractions $(<0.053 \mathrm{~mm})(\mathrm{A})$, soil organic carbon (SOC) concentration of soil aggregation and bulk (B), and aggregation stability (C) in soils following various fertilizations. The different lowercase letters above the columns indicate a meaningful difference based on one-way ANOVA with Duncan test at the level of $P<0.05$. Note: WSA, water-stable aggregates; MWD, mean weight diameter. 

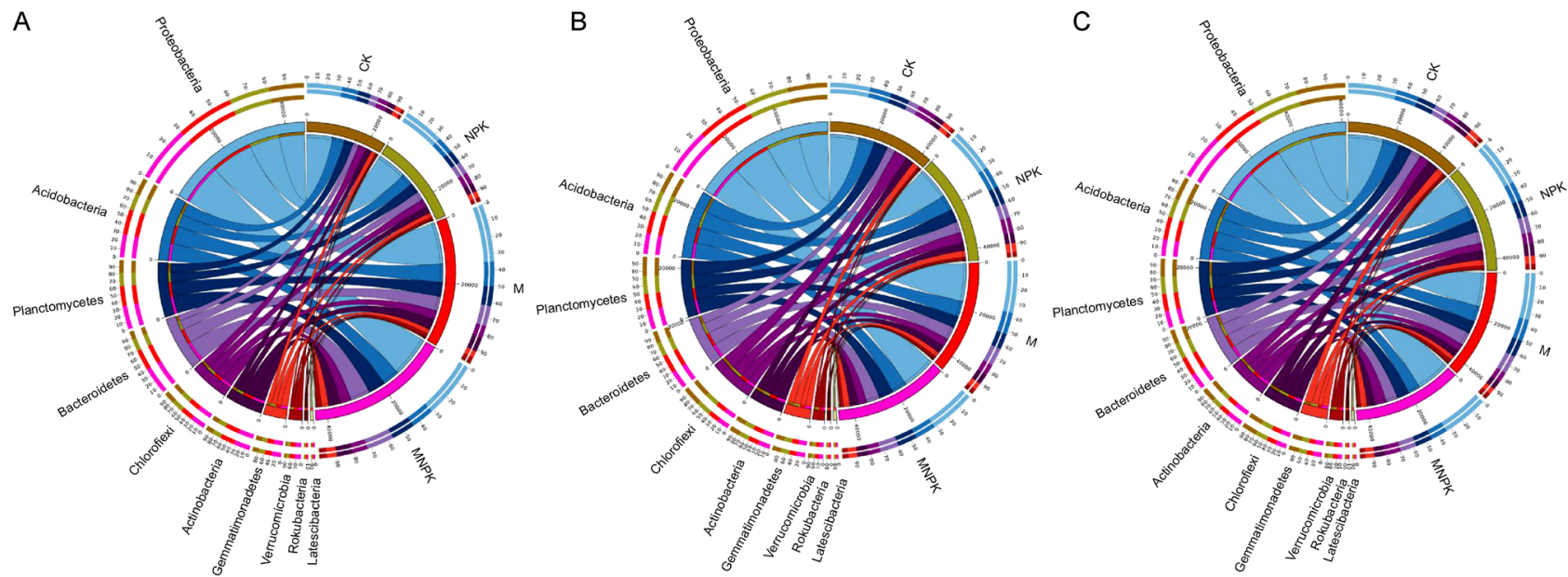

Fig. 2 Distribution of ten most abundant bacterial in macroaggregate (A), microaggregate (B) and, silt + clay fractions (C). The data was visualized via Circos software (http://circos.ca/). The length of the bars of each sample on the outer-ring represented the percentage of phyla in each sample.
Color: $\square$ CK $\square$ NPK $\square \mathrm{M} \square$ MNPK
A Symbol: $O>0.25 \mathrm{~mm} \diamond 0.053-0.25 \mathrm{~mm} \star 0.053 \mathrm{~mm} \quad$ B
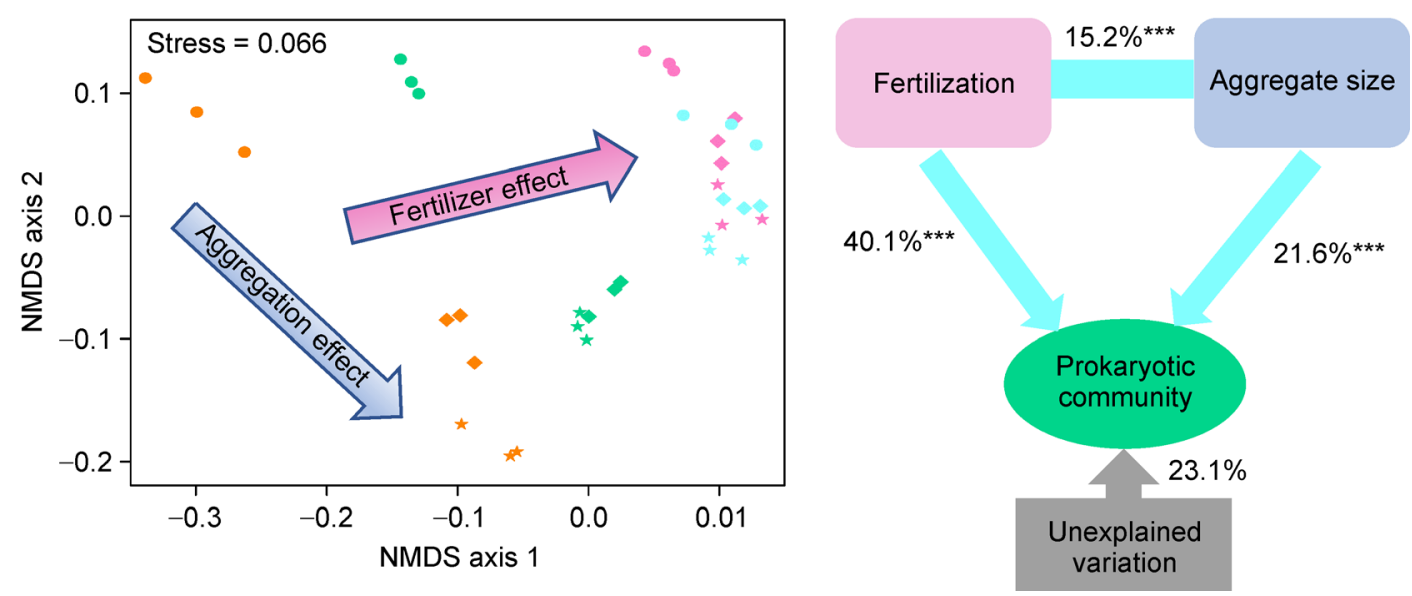

Fig. 3 Nonmetric multidimensional scaling (NMDS) analysis of communities (A). Different colors represent different treatments, and different symbols indicate different aggregate size classes. Permutational multivariate analysis of variance (PERMANOVA) comparing the main and interacting effects of fertilization and aggregate size on the community composition of bacteria (B) (999 permutations). Stars denote significant differences at $P<0.001$ probability levels $\left.{ }^{(* *}\right)$.

which, the bacterial communities of organic fertilizer-treated soils (M and MNPK) were grouped together and clearly separated from those of inorganic fertilizer-treated soils (NPK). PERMANOVA analysis further confirmed that fertilization and aggregate size, as the two most significant variables, explained $40.1 \%(P<0.01)$ and $21.6 \%(P<0.01)$ of variance in prokaryotic community structure (Fig. 3B). The interactions between the two variables explained $15.2 \%(P<0.01)$ of variance in bacterial community patterns.

\subsection{Biomarker microbes in soil aggregates}

LEFSe analysis was used to identify differentially abundant features from phylum to genus levels that evidently responded to fertilizations in all aggregate fractions (Fig. 4). In macroaggregates, 60 biomarkers with 23, 12, 9 and 16 in CK, NPK, $\mathrm{M}$ and MNPK soils, respectively, were statistically and significantly enriched (Fig. 4A). The phyla Chloroflexi, Acidobacteria, Gemmatimonadetes and Cyanobacteria were enriched in $\mathrm{CK}$ soils. The phylum Proteobacteria was enriched in NPK-treated soils. The phyla Bacteroidetes, Actinobacteria and Verrucomicrobia were remarkably abundant in MNPK-treated soils. In microaggregates, 59 biomarkers, with 26, 5, 15 and 13 in CK, NPK, M and MNPK soils, respectively, were predominant (Fig. 4B). The phyla Chloroflexi, Planctomycetes, Gemmatimonadetes and Cyanobacteria were significantly enriched in CK soils. The phylum Acidobacteria was evidently abundant in NPK-treated soils. 
A

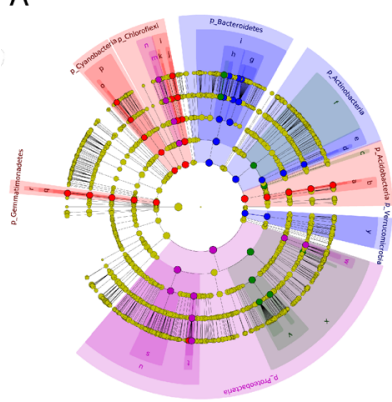

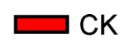

B

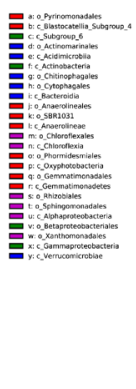

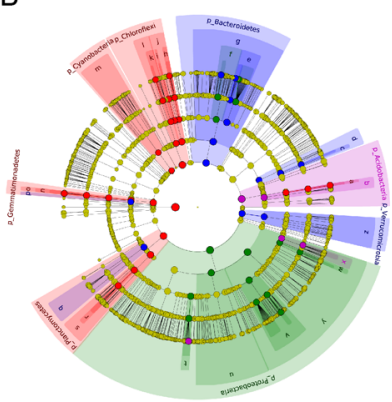

MNPK

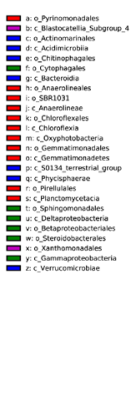

C

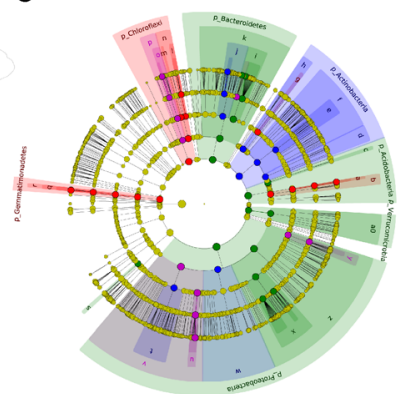

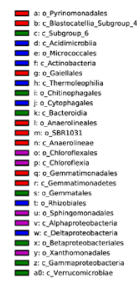

Fig. 4 LEfSe cladograms showing taxa with different abundance values. Taxonomic cladogram obtained from LEfSe analysis of bacterial 16S rRNA sequences in macroaggregate (A), microaggregate (B) and, silt + clay fractions (C). Only taxa meeting an LDA significance threshold of 3.5 for bacterial communities are shown. Seven rings of the cladogram stand for domain (innermost), phylum, class, order, family, genus, and species (outermost), respectively.

The phylum Proteobacteria was significantly enriched in $\mathrm{M}$ treated soils. The phyla Bacteroidetes and Verrucomicrobia were predominant in MNPK-treated soils. In silt and clay fractions, 52 biomarkers with 15, 11, 16 and 10 clades in CK, NPK, M, and MNPK soils, respectively, were predominant (Fig. 4C). The phyla Chloroflexi and Gemmatimonadetes were predominant in $\mathrm{CK}$ soils. The phyla Proteobacteria, Bacteroidetes, Acidobacteria, and Verrucomicrobia were predominant in M-treated soils. The phylum Actinobacteria was predominant in MNPK-treated soils.

\subsection{Network analysis of bacterial communities}

Network analysis at the phylum level was conducted to elucidate the complexity and interactions between bacterial phylotypes across all the aggregates (Fig. 5). The network of bacterial communities in each aggregate fraction exhibited distinct co-occurrence patterns. Generally, the number of correlations in the macroaggregates was substantially higher than in microaggregates, followed by silt + clay (Fig. 5; Table 1). The overall networks consisted of 2687 close associations (edges), 149 nodes with a clustering coefficient of 0.635 in macroaggregates, 874 edges and 133 nodes with a clustering coefficient of 0.496 in microaggregates, and 797 edges, 139 nodes with a clustering coefficient of 0.529 in silt + clay (Table 1). The proportion of negative links in the soil aggregate fractions followed the order of [ $<0.053 \mathrm{~mm}]>[0.053-0.25 \mathrm{~mm}]>[>0.25 \mathrm{~mm}]$ regardless of fertilization treatments. OTUs with highest degree, highest closeness centrality, and lowest betweenness centrality were selected as potential keystone taxa (Banerjee et al., 2018). It was found that Sphingomonadaceae and Burkholderiaceae, affiliated with Proteobacteria, were the keystone taxa in macroaggregate and microaggregate, respectively, while Chitinophagaceae grouped within Bacteroidetes, formed the keystone taxa in silt + clay fractions (Table 2).

\section{Discussion}

\subsection{Effects of fertilizations on soil aggregation and SOC stock}

Our work provides solid evidence that, after an experiment of 39 years, fertilization largely stimulated soil aggregation and
A

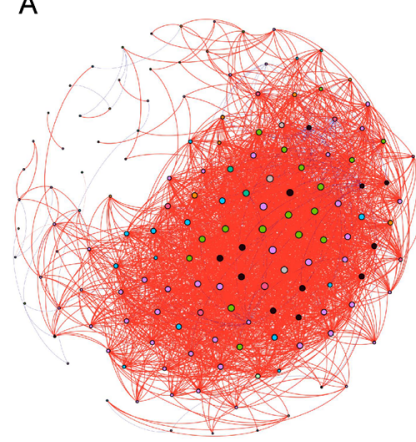

B

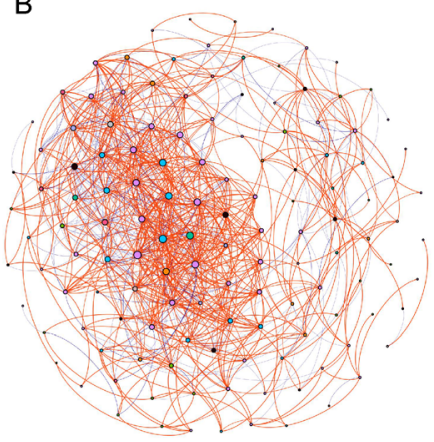

C

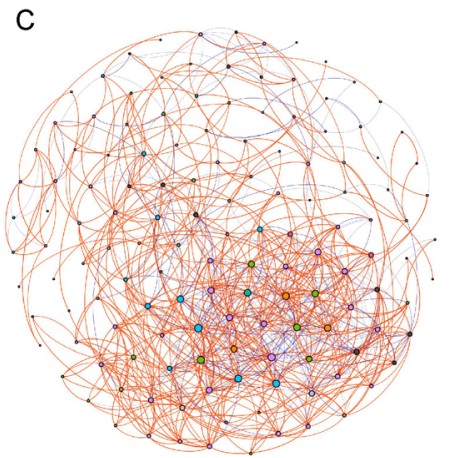

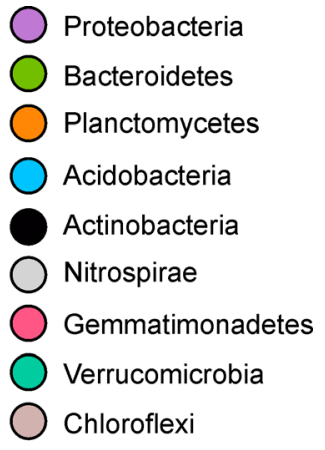

Fig. 5 Network analysis revealing bacterial co-occurrence in macroaggregates (A), microaggregates (B) and, silt + clay fractions $(C)$ in response to four fertilization treatments. Red and blue lines represent significant strong positive $(r>0.75)$ and negative $(r<-0.75)$ linear relationships. Colored nodes signify corresponding OTUs assigned to major phyla. The size of each node is proportional to the number of connections (that is, degree). 
Table 1 Network parameters.

\begin{tabular}{llllllll}
\hline Network & Nodes/edges & $\begin{array}{l}\text { Average } \\
\text { degree }\end{array}$ & $\begin{array}{l}\text { Network } \\
\text { density }\end{array}$ & $\begin{array}{l}\text { Characteristic } \\
\text { path length }\end{array}$ & $\begin{array}{l}\text { Network } \\
\text { diameter }\end{array}$ & $\begin{array}{l}\text { Modularity } \\
\text { Clustering } \\
\text { coefficient }\end{array}$ \\
\hline Macroaggregates & $149 / 2687$ & 36.067 & 0.244 & 2.381 & 8 & 0.242 & 0.635 \\
Microaggregates & $133 / 874$ & 13.143 & 0.1 & 3.17 & 10 & 1.035 & 0.496 \\
Silt and clay & $139 / 797$ & 11.468 & 0.083 & 3.295 & 9 & 1.389 & 0.529 \\
\hline
\end{tabular}

Table 2 Network features and taxonomy of the top five keystone taxa. OTUs with highest degree, highest closeness centrality, and lowest betweenness centrality were selected as keystone taxa.

\begin{tabular}{|c|c|c|c|c|c|c|}
\hline \multirow[t]{2}{*}{ Aggregate fraction } & \multirow[t]{2}{*}{ OTU ID } & \multicolumn{3}{|l|}{ Network features } & \multicolumn{2}{|l|}{ Taxonomy } \\
\hline & & Betweenness centrality & Closeness centrality & Degree & Phylum & family \\
\hline \multirow[t]{5}{*}{ Macroaggregates } & OTU_19 & 0.009 & 0.274 & 84 & Proteobacteria & Sphingomonadaceae \\
\hline & OTU_28 & 0.016 & 0.273 & 82 & Bacteroidetes & Saprospiraceae \\
\hline & OTU_42 & 0.024 & 0.274 & 81 & Proteobacteria & Uncultured \\
\hline & OTU_276 & 0.015 & 0.271 & 78 & Gemmatimonadetes & Gemmatimonadaceae \\
\hline & OTU_13 & 0.011 & 0.269 & 77 & Bacteroidetes & Chitinophagaceae \\
\hline \multirow[t]{5}{*}{ Microaggregates } & OTU_92 & 0.037 & 0.233 & 42 & Proteobacteria & Burkholderiaceae \\
\hline & OTU_105 & 0.041 & 0.234 & 42 & Bacteroidetes & Chitinophagaceae \\
\hline & OTU_52 & 0.031 & 0.227 & 39 & Chloroflexi & $A 4 b$ \\
\hline & OTU_211 & 0.021 & 0.228 & 38 & Proteobacteria & Sphingomonadaceae \\
\hline & OTU_61 & 0.024 & 0.228 & 37 & Bacteroidetes & Hymenobacteraceae \\
\hline \multirow[t]{5}{*}{ Silt and clay } & OTU_13 & 0.040 & 0.416 & 35 & Bacteroidetes & Chitinophagaceae \\
\hline & OTU_429 & 0.045 & 0.407 & 33 & Acidobacteria & Uncultured \\
\hline & OTU_78 & 0.047 & 0.411 & 33 & Proteobacteria & Steroidobacteraceae \\
\hline & OTU_61 & 0.034 & 0.406 & 32 & Bacteroidetes & Hymenobacteraceae \\
\hline & OTU_14 & 0.018 & 0.400 & 31 & Bacteroidetes & Microscillaceae \\
\hline
\end{tabular}

enhanced soil carbon stock. As revealed by the results, fertilization, especially organic fertilizations ( $\mathrm{M}$ and $\mathrm{MN}$ ), could clearly $(P<0.05)$ increase proportion of macroaggregates, aggregate stability and SOC content (Fig. 1). These results agreed well with previous findings that manure addition evidently increased SOC by enhancing aggregation (Lin et al., 2019). Notably, the SOC content was increased with the increase of soil aggregate size (Fig. 1B), which was in stark contrast with previous findings that organic amendments increased the SOC concentration the most in the microaggregates, based on the dry-sieving approach (Wang et al., 2021). This discrepancy might arise from the fact that wet sieving could lead to a loss of total $\mathrm{C}$ or total $\mathrm{N}$, especially for soil fractions $<250 \mu \mathrm{m}$ in comparison to dry sieving (Sainju, 2006). Formation and stabilization of water stable macroaggregate were largely related to SOC (Yang et al., 2007). High aggregation levels reduce the activity of enzymes involved in the degradation of labile SOM, thus protecting the SOM from decomposing and increasing SOC accumulation (Lin et al., 2019). On the other hand, the increase of SOC in turn stimulates formation and stabilization of macroaggregates, as SOC is admittedly important in binding soil particles into macroaggregates (Six et al., 2000). Therefore, formation and stability of soil aggregate and SOC content interacted. Fertilization (especially manure) provided functional groups (aromatic, heterocyclic structures and carboxyl groups) to boost formation and stabilization of soil macroaggregates, which in turn boosted SOC content.

The SOC content in bulk soils was substantially increased by the long-term organic fertilizations (Fig. 1B), which was due to a heightened input of organic matter through manure and root biomass through enhanced growth of crops (He et al., 2018b). However, the 39-year chemical fertilizations (NPK) slightly increased SOC in bulk soils, which was in agreement with the findings of $\mathrm{He}$ et al. (2015), but diverged from those of Maillard et al. (2015) and He et al. (2018b). This discrepancy could be attributed to a lower $C$ input in the present study as the aboveground crop residues were removed from the field. Meanwhile, the SOC contents in bulk soils were notably lower than those in macroaggregates and microaggregates, which 
suggested that aggregates fractions were more effective than bulk soils for capturing SOC to fertilizations.

4.2 Effects of fertilizations on soil bacterial communities at aggregate scale

NMDS analysis revealed that aggregate size and fertilization strategies meaningfully impacted prokaryotic community structure and diversity (Fig. 3A). PERMANOVA analysis further demonstrated that fertilization practices exhibited more influence than aggregate size in shaping microbial community patterns (Fig. 3B). Briar et al. (2011) previously proposed that resource availability rather than the aggregate sizes shaped bacterial communities. Increased SOC in macroaggregates translated into increased food availability for microbial communities. Fertilizations, especially organic fertilizers (M and MNPK) primarily facilitated the growth of copiotrophic groups, e.g., Proteobacteria and Bacteroidetes, which comprise many types of functional genes. These bacteria are involved in labile substrate degradation and prefer high $C$ availability, and could stimulate soil nutrient cycling and provide nutrients for crops (Xun et al., 2016; Wolińska et al., 2017). On the contrary, fertilizations, especially manure addition, were not conducive to the growth of Chloroflexi and Gemmatimonadetes in all aggregates (Fig. 2). The remarkable decrease of Chloroflexi in fertilized soils resulted from the prevalence of Chloroflexi in oligotrophic environments (Janssen 2006; Will et al., 2010). It has been reported that the relative abundance of Gemmatimonadetes is not related to land management but is inversely correlated to soil moisture (DeBruyn et al., 2011). Thus, the remarkable depression of Gemmatimonadetes under fertilizations could be due to the increase of soil moisture after fertilizations (DeBruyn et al., 2011). Meanwhile, phylum Gemmatimonadetes is usually known to suppress disease and promote plant growth (Li et al., 2017). The obvious decrease of Gemmatimonadetes by fertilizations suggested that fertilizer application could decrease the soil's intrinsic disease-resistance. Therefore, after the 39-year fertilizations, the bacterial communities transitioned from dominance by slow-growing oligotrophic groups (e.g., Chloroflexi) to that by fast-growing copiotrophic groups (e.g., Proteobacteria and Bacteroidetes) across all aggregate sizes.

The LEfSe analysis showed that the numbers of biomarkers in NPK-treated soils were clearly lower than those in organic fertilized soils (Fig. 4). Combined with the results that the proportion of biomarkers under $M$ treatments was substantially increased with a decrease in aggregate size. We proposed that bacterial communities were more sensitive to organic fertilization than to mineral fertilization at aggregate scale, consistent with previous results for bulk soils (Pan et al., 2020). Previously, Liao et al. (2018) proposed that bacterial communities were driven by chemical fertilization, as they were closely dependent on the shifts of soil phosphorus. More studies have reported that organic treatments greatly alter the microbial community structure mainly by changing the SOC content (Ding and Han, 2014; Wang et al., 2021).

4.3 Effects of soil aggregate sizes on bacterial community patterns

Soil prokaryotic community patterns were notably altered by aggregate sizes (Fig. 2), which was largely related to the resource availability induced by heterogeneity of physicochemical properties. Soil aggregates had complex pore network, with various levels of SOC, water and oxygen availability (Garbout et al., 2013; Rillig et al., 2017), which could influence soil prokaryotic communities. Macroaggregates generally had more labile substrates, while smaller aggregates contained more recalcitrant SOM (Lin et al., 2019). Overall, Proteobacteria, Acidobacteria, Planctomycetes, Bacteroidetes, Chloroflexi, Actinobacteria, and Gemmatimonadetes were the dominant bacterial groups in all aggregate fractions (Fig. 2), which were consistent with our previous results using bulk soils at the same site (Pan et al., 2020). Notably, the proportion of Proteobacteria was substantially higher in macroaggregates than those in small aggregates and was remarkably higher in fertilized soils than in CK soils (Fig. 2). These findings were generally consistent with Trivedi et al. (2017) who isolated soil aggregates based on the optimal moisture approach, and reported that bacterial taxa were faster growing and more copiotrophic, thus being favored by large aggregates, while slow growing and more oligotrophic taxa dominated micro-aggregates.

Additionally, the numbers of biomarkers in macroaggregates and microaggregates were 60 and 59, respectively, which were evidently higher than in silt + clay (52 biomarkers). This indicated that the bacterial communities in silt + clay fractions were less sensitive to long-term fertilizations than those in larger aggregates (Yang et al., 2007; Dai et al., 2019). The lower vitality of bacterial communities in silt and clay fraction also explained why the nutrient turnover was much slower in the silt + clay aggregate size class than in larger aggregates.

The LEfSe analysis revealed that phylum Planctomycetes was enriched exclusively in 0.053-0.25 mm microaggregates, phylum Cyanobacteria was enriched in $>0.25 \mathrm{~mm}$ macroaggregates and $0.053-0.25 \mathrm{~mm}$ microaggregates, while phylum Actinobacteria was only enriched in $>0.25 \mathrm{~mm}$ macroaggregates and $<0.053 \mathrm{~mm}$ silt + clay. Previous studies underscored that some members of the phylum Planctomycetes participate in degradation of plant detritus and cellulose by encoding enzymes, which facilitates the $\mathrm{N}$ mineralisation of straw (Schellenberger et al., 2010; Guo et al., 2021). It has been reported that Cyanobacteria can promote aggregate formation, by forming photosynthetic microbiotic crusts, thus improving the structural stability of topsoil, in temperate croplands (Crouzet et al., 2019). Actinobacteria genera are believed to play a dominant role in degradation of hemicellulose and cellulose, which may enhance the decomposition process of residues (Wang et al., 2016). Therefore, our results confirmed that different aggregate sizes provide 
spatially heterogeneous microhabitats, and support the colonization of distinct microbial communities, which in turn result in distinct metabolic activities and functions.

The network topological patterns were distinct for different aggregate sizes (Fig. 5). The total nodes and links, average degree and network density were increased with the increase of aggregate sizes, while characteristic path length followed the opposite trend (Table 1; Fig. 5). This indicated that the network of macroaggregates incorporated a substantially higher number of close correlations than those of microaggregate and silt + clay (Fig. 5), suggesting that soil microbial communities of macroaggregates were more complexly connected. The aggregate size class with high complexity of bacterial groups could provide biological buffering and thus was potentially stable after 39-year fertilizations. The less stable of network in smaller aggregates was due to the lack of physical protection for microbes among small particles (Dai et al., 2019). Meanwhile, the modularity in macroaggregate was significantly lower than that in smaller fractions (Table 1), indicating that macroaggregate had higher niche overlap than microaggregate and silt + clay fractions. The high symbiotic relationships of the network pattern in macroaggregates resulted from the stronger cooperative and trophic interactions among bacterial communities induced by the higher resource availability and lower nutritional stress (Briar et al., 2011; Morriën et al., 2017). The complex interactions also indicated more rapid $\mathrm{C}$ and $\mathrm{N}$ turnover rates in macroaggregates than those in microaggregates and silt + clay. Notably, the proportions of negative relationships were increased with decrease of aggregate sizes. This could also be explained by the resource availability as labile SOC content decreased with a decrease in aggregate size, where intensified competition among microbes arose, due to the less favorable conditions. On the other hand, the network dominated with positive interactions in macroaggregates has the potential to facilitate soil function. Therefore, these results highlight the paramount importance of the soil aggregation in orchestrating microbial communities to enhance ecological functions.

\section{Conclusions}

Generally speaking, these findings suggested that the 39-year fertilizations, especially organic fertilizations, could improve soil quality by improving soil physical structure and regulating soil microbial community compositions. Fertilization regimes and aggregate sizes were the two main factors in modifying the bacterial community structures. The bacterial community structures were more sensitive to organic fertilizations than to inorganic fertilizations, and could be transitioned from slowgrowing oligotrophic groups (e.g., Chloroflexi) dominance to fast-growing copiotrophic groups (e.g., Proteobacteria and Bacteroidetes) dominance across all aggregate sizes in the long term fertilized soils. The network of macroaggregates incorporated a substantially higher number of close correlations than those of microaggregate and silt + clay, and indicated that the interactions among microbes, niche overlap and $\mathrm{C}$ turnover were much higher in macroaggregates than in smaller aggregates. These results could deepen our understanding of microbiome structure and functioning and their underlying processes in driving soil quality and sustainability of agroecosystems.

\section{Acknowledgments}

This work was funded by the National Natural Science Foundation of China (42007076), the Shandong Provincial Natural Science Foundation (ZR2020QD116 and ZR2019BD032), the China Postdoctoral Science Foundation (2020T130387 and 2019M652448).

\section{Conflict of interest}

The authors declare that they have no conflict of interest.

\section{References}

Ashagrie, Y., Zech, W., Guggenberger, G., Mamo, T., 2007. Soil aggregation, and total and particulate organic matter following conversion of native forests to continuous cultivation in Ethiopia. Soil \& Tillage Research 94, 101-108.

Bailey, V.L., Fansler, S.J., Stegen, J.C., McCue, L.A., 2013. Linking microbial community structure to $\beta$-glucosidic function in soil aggregates. ISME Journal 7, 2044-2053.

Banerjee, S., Schlaeppi, K., van der Heijden, M.G., 2018. Keystone taxa as drivers of microbiome structure and functioning. Nature Reviews. Microbiology 16, 567-576.

Bastian, M., Heymann, S., Jacomy, M., 2009. Gephi: an open source software for exploring and manipulating networks. Third International ICWSM Conference, 361-362.

Biederbeck, V.O., Curtin, D., Bouman, O.T., Campbell, C.A., Ukrainetz, H., 1996. Soil microbial and biochemical properties after ten years of fertilization with urea and anhydrous ammonia. Canadian Journal of Soil Science 76, 7-14.

Blanco-Canqui, H., Schlegel, A.J., 2013. Implications of inorganic fertilization of irrigated corn on soil properties: Lessons learned after 50 years. Journal of Environmental Quality 42, 861-871.

Bottinelli, N., Angers, D.A., Hallaire, V., Michot, D., Le Guillou, C., Cluzeau, D., Heddadj, D., Menasseri-Aubry, S., 2017. Tillage and fertilization practices affect soil aggregate stability in a Humic Cambisol of Northwest France. Soil \& Tillage Research 170, 14 17.

Briar, S.S., Fonte, S.J., Park, I., Six, J., Scow, K., Ferris, H., 2011. The distribution of nematodes and soil microbial communities across soil aggregate fractions and farm management systems. Soil Biology \& Biochemistry 43, 905-914.

Crouzet, O., Consentino, L., Pétraud, J.P., Marrauld, C., Aguer, J.P., Bureau, S., Le Bourvellec, C., Touloumet, L., Berard, A., 2019. Soil photosynthetic microbial communities mediate aggregate stability: influence of cropping systems and herbicide use in an agricultural soil. Frontiers in Microbiology 10, 1319. 
DeBruyn, J. M., Nixon, L. T., Fawaz, M. N., Johnson, A. M., \& Radosevich, M., 2011. Global biogeography and quantitative seasonal dynamics of Gemmatimonadetes in soil. Applied and Environmental Microbiology 77, 6295-6300

Dai, H., Zang, H., Zhao, Y., Qian, X., Liu, K., Wang, D., Hao, J., Chen, Y., Sui, P., 2019. Linking bacterial community to aggregate fractions with organic amendments in a sandy soil. Land Degradation \& Development 30, 1828-1839.

DeBruyn, J.M., Nixon, L.T., Fawaz, M.N., Johnson, A.M., Radosevich, M., 2011. Global biogeography and quantitative seasonal dynamics of Gemmatimonadetes in soil. Applied and Environmental Microbiology 77, 6295-6300.

Ding, X., Han, X., 2014. Effects of long-term fertilization on contents and distribution of microbial residues within aggregate structures of a clay soil. Biology and Fertility of Soils 50, 549-554.

Elliott, E.T., 1986. Aggregate structure and carbon, nitrogen, and phosphorus in native and cultivated soils. Soil Science Society of America Journal 50, 627-633.

Fierer, N., 2017. Embracing the unknown: disentangling the complexities of the soil microbiome. Nature Reviews. Microbiology 15, 579-590.

Feng, H., Wang, S., Gao, Z., Wang, Z., Ren, X., Hu, S., \& Pan, H., 2019. Effect of land use on the composition of bacterial and fungal communities in saline-sodic soils. Land Degradation \& Development 30, 1851-1860

Garbout, A., Munkholm, L. J., \& Hansen, S. B., 2013. Tillage effects on topsoil structural quality assessed using $X$-ray $\mathrm{CT}$, soil cores and visual soil evaluation. Soil and Tillage Research 128, 104-109

Ghosh, B.N., Meena, V.S., Singh, R.J., Alam, N.M., Patra, S., Bhattacharyya, R., Sharma, N.K., Dadhwal, K.S., Mishra, P.K., 2019. Effects of fertilization on soil aggregation, carbon distribution and carbon management index of maize-wheat rotation in the north-western Indian Himalayas. Ecological Indicators 105, 415424.

Guo, T., Zhang, Q., Ai, C., He, P., Zhou, W., 2021. Microbial utilization of rice root residue-derived carbon explored by DNA stable-isotope probing. European Journal of Soil Science 72, 460-473.

Gupta, V.V., Germida, J.J., 2015. Soil aggregation: Influence on microbial biomass and implications for biological processes. Soil Biology \& Biochemistry 80, A3-A9.

Han, S., Delgado-Baquerizo, M., Luo, X., Liu, Y., Van Nostrand, J.D., Chen, W., Zhou, J., Huang, Q., 2021. Soil aggregate sizedependent relationships between microbial functional diversity and multifunctionality. Soil Biology \& Biochemistry 154, 108143.

Han, S., Luo, X., Hao, X., Ouyang, Y., Zeng, L., Wang, L., Wen, S., Wang, B., Van Nostrand, J.D., Chen, W., Zhou, J., Huang, Q., 2020. Microscale heterogeneity of the soil nitrogen cycling microbial functional structure and potential metabolism. Environmental Microbiology 23, 1199-1209 .

Han, S., Xiong, X., Luo, X., Zeng, L., Wei, D., Chen, W., Huang, Q., 2018. Fertilization rather than aggregate size fractions shape the nitrite-oxidizing microbial community in a Mollisol. Soil Biology \& Biochemistry 124, 179-183.

He, Y., Xu, C., Gu, F., Wang, Y., Chen, J., 2018a. Soil aggregate stability improves greatly in response to soil water dynamics under natural rains in long-term organic fertilization. Soil \& Tillage
Research 184, 281-290.

He, Y.T., He, X.H., Xu, M.G., Zhang, W.J., Yang, X.Y., Huang, S.M., 2018b. Long-term fertilization increases soil organic carbon and alters its chemical composition in three wheat-maize cropping sites across central and south China. Soil \& Tillage Research 177, 79 87.

He, Y.T., Zhang, W.J., Xu, M.G., Tong, X.G., Sun, F.X., Wang, J.Z., Huang, S.M., Zhu, P., He, X.H., 2015. Long-term combined chemical and manure fertilizations increase soil organic carbon and total nitrogen in aggregate fractions at three typical cropland soils in China. Science of the Total Environment 532, 635-644.

Janssen, P.H., 2006. Identifying the dominant soil bacterial taxa in libraries of $16 \mathrm{~S}$ rRNA and 16S rRNA genes. Applied and Environmental Microbiology 72, 1719-1728.

Jiang, M., Wang, X., Liusui, Y., Han, C., Zhao, C., Liu, H., 2017. Variation of soil aggregation and intra-aggregate carbon by longterm fertilization with aggregate formation in a grey desert soil. Catena 149, 437-445.

Jiang, Y., Jin, C., Sun, B., 2014. Soil aggregate stratification of nematodes and ammonia oxidizers affects nitrification in an acid soil. Environmental Microbiology 16, 3083-3094.

Jiang, Y., Sun, B., Jin, C., Wang, F., 2013. Soil aggregate stratification of nematodes and microbial communities affects the metabolic quotient in an acid soil. Soil Biology \& Biochemistry 60, 1-9.

Li, F., Chen, L., Zhang, J., Yin, J., Huang, S., 2017. Bacterial community structure after long-term organic and inorganic fertilization reveals important associations between soil nutrients and specific taxa involved in nutrient transformations. Frontiers in Microbiology 8, 1-12.

Li, P.P., Han, Y.L., He, J.Z., Zhang, S.Q., Zhang, L.M., 2019. Soil aggregate size and long-term fertilization effects on the function and community of ammonia oxidizers. Geoderma 338, 107-117.

Liao, H., Zhang, Y., Wang, K., Hao, X., Chen, W., Huang, Q., 2020. Complexity of bacterial and fungal network increases with soil aggregate size in an agricultural Inceptisol. Applied Soil Ecology 154, 103640.

Liao, H., Zhang, Y., Zuo, Q., Du, B., Chen, W., Wei, D., Huang, Q., 2018. Contrasting responses of bacterial and fungal communities to aggregate-size fractions and long-term fertilizations in soils of northeastern China. Science of the Total Environment 635, 784792.

Lin, Y., Ye, G., Kuzyakov, Y., Liu, D., Fan, J., Ding, W., 2019. Longterm manure application increases soil organic matter and aggregation, and alters microbial community structure and keystone taxa. Soil Biology \& Biochemistry 134, 187-196.

Liu, M., Wang, C., Liu, X., Lu, Y., Wang, Y., 2020. Saline-alkali soil applied with vermicompost and humic acid fertilizer improved macroaggregate microstructure to enhance salt leaching and inhibit nitrogen losses. Applied Soil Ecology 156, 103705

Morriën, E., Hannula, S.E., Snoek, L.B., Helmsing, N.R., Zweers, H., De Hollander, M., Soto, R.L., Bouffaud, M.L., Buée, M., Dimmers, W., Duyts, H., Geisen, S., Girlanda, M., Griffiths, R.I., Jørgensen, H.B., Jensen, J., Plassart, P., Redecker, D., Schmelz, R.M., Schmidt, O., Thomson, B.C., Tisserant, E., Uroz, S., Winding, A., Bailey, M.J., Bonkowski, M., Faber, J.H., Martin, F., Lemanceau, P., de Boer, W., van Veen, J.A., van der Putten, W.H., 2017. Soil 
networks become more connected and take up more carbon as nature restoration progresses. Nature Communications 8, 14349.

Mougi, A., Kondoh, M., 2012. Diversity of interaction types and ecological community stability. Science 337, 349-351.

Muruganandam, S., Israel, D.W., Robarge, W.P., 2010. Nitrogen transformations and microbial communities in soil aggregates from three tillage systems. Soil Science Society of America Journal 74, $120-129$

Maillard, É., Angers, D. A., Chantigny, M., Bittman, S., Rochette, P., Lévesque, G., Hunt, D., Parent, L. É., 2015. Carbon accumulates in organo-mineral complexes after long-term liquid dairy manure application. Agriculture, Ecosystems \& Environment 202, 108-119

Mustafa, A., Minggang, X., Ali Shah, S.A., Abrar, M.M., Nan, S., Baoren, W., Zejiang, C., Saeed, Q., Naveed, M., Mehmood, K., Núñez-Delgado, A., 2020. Soil aggregation and soil aggregate stability regulate organic carbon and nitrogen storage in a red soil of southern China. Journal of Environmental Management 270, 110894.

Neutel, A.M., Heesterbeek, J.A.P., Van De Koppel, J., Hoenderboom, G., Vos, A., Kaldeway, C., Berendse, F., De Ruiter, P.C., 2007. Reconciling complexity with stability in naturally assembling food webs. Nature 449, 599-602.

Pan, H., Chen, M., Feng, H., Wei, M., Song, F., Lou, Y., Cui, X., Wang, H., Zhuge, Y., 2020. Organic and inorganic fertilizers respectively drive bacterial and fungal community compositions in a fluvo-aquic soil in northern China. Soil \& Tillage Research 198, 104540.

Rillig, M.C., Muller, L.A., Lehmann, A., 2017. Soil aggregates as massively concurrent evolutionary incubators. ISME Journal 11 1943-1948.

Sainju, U.M., 2006. Carbon and nitrogen pools in soil aggregates separated by dry and wet sieving methods. Soil Science 171, 937 949.

Schellenberger, S., Kolb, S., Drake, H.L., 2010. Metabolic responses of novel cellulolytic and saccharolytic agricultural soil Bacteria to oxygen. Environmental Microbiology 12, 845-861.

Six, J., Elliott, E.T., Paustian, K., 1999. Aggregate and soil organic matter dynamics under conventional and no-tillage systems. Soil Science Society of America Journal 63, 1350-1358.

Six, J., Elliott, E.T., Paustian, K., 2000. Soil structure and soil organic matter II. A normalized stability index and the effect of mineralogy. Soil Science Society of America Journal 64, 1042-1049.

Stone, D.L., Whitney, D., Janssen, K.A., Long, J.H., 1991. Soil properties after twenty years of fertilization with different nitrogen sources. Soil Science Society of America Journal 55, 1097-1100.

Tiemann, L.K., Grandy, A.S., Atkinson, E.E., Marin-Spiotta, E., McDaniel M.D., 2015. Crop rotational diversity enhances belowground communities and functions in an agroecosystem. Ecology Letters $18,761-771$.
Trivedi, P., Rochester, I.J., Trivedi, C., Van Nostrand, J.D., Zhou, J., Karunaratne, S., Anderson, I.C., Singh, B.K., 2015. Soil aggregate size mediates the impacts of cropping regimes on soil carbon and microbial communities. Soil Biology \& Biochemistry 91, 169-181.

Trivedi, P., Delgado-Baquerizo, M., Jeffries, T.C., Trivedi, C., Anderson, I.C., Lai, K., McNee, M., Flower, K., Pal Singh, B., Minkey, D., Singh, B.K., 2017. Soil aggregation and associated microbial communities modify the impact of agricultural management on carbon content. Environmental Microbiology 19, 30703086.

Wang, C., Dong, D., Wang, H., Müller, K., Qin, Y., Wang, H., Wu, W., 2016. Metagenomic analysis of microbial consortia enriched from compost: new insights into the role of Actinobacteria in lignocellulose decomposition. Biotechnology for Biofuels 29, 22.

Wang, M.C., Yang, C.H., 2003. Type of fertilizer applied to a paddyupland rotation affects selected soil quality attributes. Geoderma 114, 93-108.

Wang, X., Bian, Q., Jiang, Y., Zhu, L., Chen, Y., Liang, Y., Sun, B., 2021. Organic amendments drive shifts in microbial community structure and keystone taxa which increase C mineralization across aggregate size classes. Soil Biology \& Biochemistry 153 , 108062.

Whalen, J.K., Chang, C., 2002. Macroaggregate characteristics in cultivated soils after 25 annual manure applications. Soil Science Society of America Journal 66, 1637-1647.

Will, C., Thürmer, A., Wollherr, A., Nacke, H., Herold, N., Schrumpf, M., Gutknecht, J., Wubet, T., Buscot, F., Daniel, R., 2010. Horizonspecific bacterial community composition of German grassland soils, as revealed by pyrosequencing-based analysis of 16S rRNA genes. Applied and Environmental Microbiology 76, 6751-6759.

Wolińska, A., Kuźniar, A., Zielenkiewicz, U., Izak, D., SzafranekNakonieczna, A., Banach, A., Błaszczyk, M., 2017. Bacteroidetes as a sensitive biological indicator of agricultural soil usage revealed by a culture-independent approach. Applied Soil Ecology 119, 128-137.

Xun, W., Zhao, J., Xue, C., Zhang, G., Ran, W., Wang, B., Shen, Q., Zhang, R., 2016. Significant alteration of soil bacterial communities and organic carbon decomposition by different long-term fertilization management conditions of extremely low-productivity arable soil in South China. Environmental Microbiology 18, 1907-1917.

Yang, Z., Singh, B.R., Hansen, S., 2007. Aggregate associated carbon, nitrogen and sulfur and their ratios in long-term fertilized soils. Soil \& Tillage Research 95, 161-171.

Young, I.M., Crawford, J.W., Nunan, N., Otten, W., Spiers, A., 2008. Microbial distribution in soils: physics and scaling. Advances in Agronomy 100, 81-121.

Zhou, H., Peng, X., Perfect, E., Xiao, T., Peng, G., 2013. Effects of organic and inorganic fertilization on soil aggregation in an Ultisol as characterized by synchrotron based X-ray micro-computed tomography. Geoderma 195-196, 23-30. 